Fetal Diagnosis and Therapy
Fetal Diagn Ther 2009;25:361-372

DOI: $10.1159 / 000236148$
Received: June 5, 2009

Accepted: June 9, 2009

Published online: September 30, 2009

\title{
Three- and Four-Dimensional Fetal Echocardiography
}

\author{
Sifa Turan Ozhan Turan Ahmet A. Baschat \\ Department of Obstetrics, Gynecology and Reproductive Sciences, University of Maryland School of Medicine, \\ Baltimore, Md., USA
}

\section{Key Words}

Fetal echocardiography - Spatiotemporal image correlation * Tomographic ultrasound imaging • Ultrasound, 3D/4D • Volume analyses $\cdot$ Volume computer-aided diagnosis

\begin{abstract}
The rapid continuing development of 3-dimensional ultrasound imaging technologies has extended capabilities of fetal cardiac scanning beyond 2-dimensional imaging technologies and now provides a wide array of analytic possibilities. The acquisition of the 3D volume information is based on initial application of 2-dimensional imaging techniques including grey scale, Doppler, Power Doppler and Bflow modalities. Once the cardiac volume is obtained a combination of postprocessing tools such as surface mode, minimal mode, transverse rendering, inversion and glass body modes allow preferential display of various features of the fetal heart. Motion display modes including cine-loop and spatiotemporal image correlation in combination with automated and semiautomated display of examination planes opens a whole new array of diagnostic possibilities in clinical practice.

Copyright $\odot 2009$ S. Karger AG, Basel
\end{abstract}

\section{Introduction}

Two-dimensional echocardiography is the gold standard for prenatal imaging of the fetal heart and situs. In order to adapt to the examination of the fetus, the pediatric examination technique had to be modified. The transverse plane for the imaging of short-axis views, and the longitudinal planes for the imaging of outflow tracts and the 4-chamber view were initially introduced to provide a simplified standard for the pediatric cardiac examination [1]. However, prenatally sonolucency of the fetal ribs and the fluid-filled lungs allows imaging of the heart and great vessels in many views that are not obtainable postnatally. Yet, the traditional approach to fetal cardiac imaging is still based on the demonstration of complex 3D relationships by a segmental documentation of the $2 \mathrm{D}$ planes [2-4]. Because the fetal heart is a moving structure and cardiac assessment typically involves evaluation of static and moving images, there were inherent limitations of $3 \mathrm{D}$ acquisition technology in the past. However, the rapid development of 3D ultrasound imaging technologies has extended capabilities of fetal cardiac scanning beyond $2 \mathrm{D}$ imaging technologies and now provides a wide array of analytic possibilities.

The principal acquisition technique for $3 \mathrm{D}$ cardiac imaging is still based on 2D ultrasound technology. However, 3D ultrasound offers post-processing capabilities which affect how tissue and blood flow are displayed in

Ahmet A. Baschat, MD

Department of Obstetrics, Gynecology and Reproductive Sciences

University of Maryland School of Medicine, 405 West Redwood Street, 4th Floor Baltimore, MD 21201 (USA)

Tel. +1 410328 8874, Fax +1 410328 1669, E-Mail abaschat@umm.edu 
1

Fig. 1. Longitudinal view of the thorax and upper abdomen. Power Doppler allows visualization of all vessels. DAo $=$ Descending aorta; $\mathrm{DV}=$ ductus venosus; $\mathrm{IVC}=$ inferior vena cava; $U V=$ umbilical vein.

Fig. 2. Aortic arch with the origin of cephalic vessels is imaged using high-definition color Doppler. BC = Brachiocephalic (innominate) artery; DAo = descending aorta; LCC = left common carotid artery; LS = left subclavian artery.

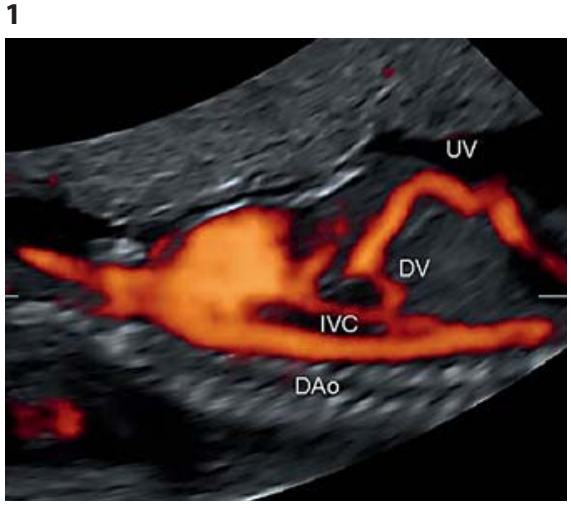

2

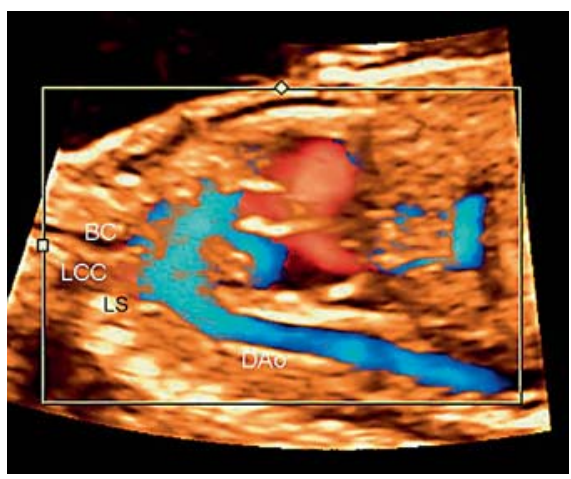

relation to each other. In addition to these post-processing capabilities, there is the possibility of manipulating ultrasound information in variable or preset planar manipulation techniques. The purpose of this review is to summarize these technical aspects and their clinical application in $3 \mathrm{D}$ and $4 \mathrm{D}$ fetal cardiac imaging.

\section{Principles of Image Acquisition}

\section{$2 D$ Imaging Considerations}

Two-dimensional imaging of the fetal heart balances spatial and temporal resolution. Spatial resolution determines the degree to which anatomic detail can be displayed and temporal resolution is an important additional factor since the fetal heart is in constant motion. Accordingly, the typical setup for cardiac imaging utilizes a single acoustic focal zone, a narrow image field and magnification to display the area of interest. In addition, a low dynamic range emphasizes the contrast between tissue and blood-filled structures. Using this setup high resolution and high frame rate imaging can be achieved with modern ultrasound equipment.

Color Doppler, power Doppler, high definition power Doppler and B-flow can be added to the grayscale image prior to 3D image acquisition. A narrow image field must be chosen to maintain a high frame rate.

\section{Vascular Imaging Modalities}

As grayscale ultrasound reflection is greatest with perpendicular insonation, and Doppler reflection is greatest at 0 -degree insonation, combination with color Doppler imaging is always a compromise between optimal grayscale versus Doppler insonation. Therefore, when grayscale and color Doppler are combined, best visualization of all cardiac structures in the 4-chamber view is obtained with the cardiac axis at approximately 45 degrees to the transducer. The persistence settings need to be low, in order to allow demonstration of phasic blood flow, and a variance mode of the color spectrum is preferable with color Doppler imaging to facilitate detection of turbulent flow. Unlike color Doppler, power Doppler is unhelpful for the determination of blood flow directionality but very useful for reconstruction of vascular trees. This feature has aided our understanding of the normal and anomalous anatomy and pathophysiology of vascular lesions [5] (fig. 1). High-definition power Doppler uses high resolution and a small sample volume to produce images with 2-color directional information. It more realistically represents the vessel size and depicts flow at a lower velocity than color or power Doppler, therefore allowing better identification of small vessels in the vascular tree [6] (fig. 2).

B-flow is a technology that images blood flow without relying on Doppler shift. The reflected amplitudes of scattering particles, such as erythrocytes in flowing blood, is imaged by a subtraction mode of 2 or 4 image vectors along one line. In this setting only moving particles are imaged and stationary structures, such as the vessel walls, are subtracted ('brightness' mode of B-flow). B-flow data is then combined with B-mode information enabling a significantly better amplitude visualization of the flow, independent of the angle between probe and centerline of the vessel. In color-coded B-flow, surrounding structures, such as the vessel wall, are added for anatomic orientation. The brightness of the color signal corresponds to the flow velocity. B-flow offers simultaneous detection and display of tissue and at the same time highresolution spatial and temporal depiction of blood flow. The discrimination of blood flow and the luminal vessel outline compared to color Doppler is much more accurate. The independence of the transmit angle and the al- 
3

Fig. 3. B-flow image of the normal heart and aortic arch, showing the brachiocephalic artery (BC), left common carotid artery (LCC) and left subclavian artery (LS) arising from the aortic arch. DAo = Descending aorta; DV = ductus venosus; $\mathrm{IVC}=$ inferior vena cava; $\mathrm{UV}=$ umbilical vein.

Fig. 4. Acquisition plane, ROI. The dimension of the ROI should include all structures to be studied. DAo = Descending aorta.
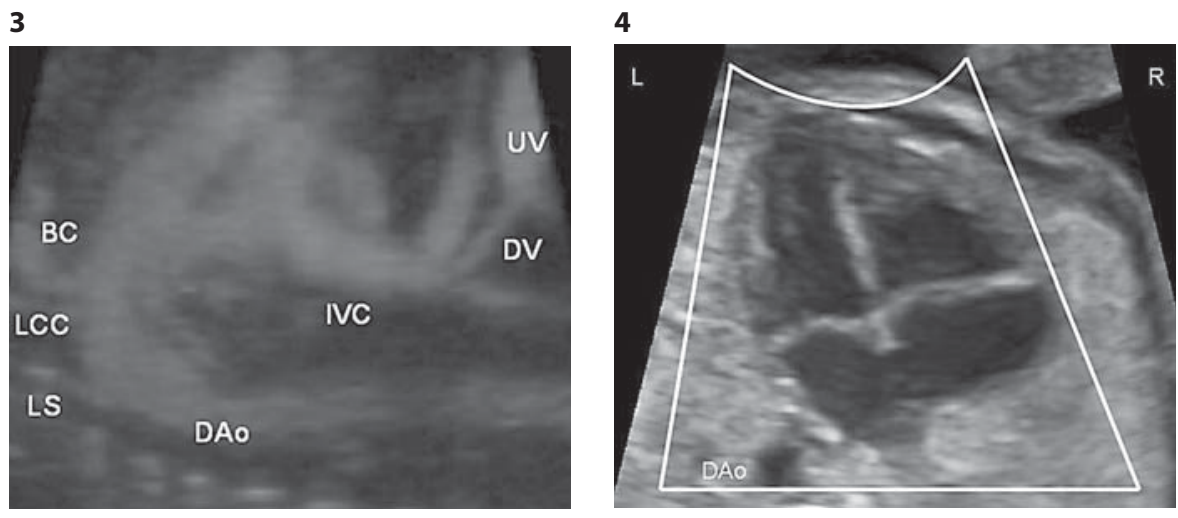

most artifact-free depiction eases the flow assessment and allows accurate assessment of vessel stenosis. One major advantage of B-flow is the lack of the color blooming or aliasing artifacts associated with Doppler techniques (fig. 3).

\section{D Imaging Considerations}

Acquisition Plane. The acquisition plane is the starting section from which the volume will be acquired, symmetrically upwards and downwards. Current 3D image acquisition is based on the sequential sampling of closely related $2 \mathrm{D}$ planes in a mechanical sweep. The image that is displayed in the A plane and any parallel plane will therefore correspond to the 2D starting image and therefore have the highest image resolution. In contrast, planes $\mathrm{B}$ and $\mathrm{C}$ are reconstructed and will have lower image resolution. The optimal acquisition plane for 3D imaging therefore corresponds to the optimal 2D examination plane, especially if color Doppler is also used. In this conventional transverse acquisition, the fetus will ideally be lying with the spine at 6 o'clock. In practice, good image quality can be obtained with any position except for spine anterior (spine between 11 o'clock and 1 o'clock), where acoustic shadowing from the vertebrae hides cardiac structures. To better visualize the interventricular septum it is also advisable to avoid an apical 4-chamber view.

Region of Interest. The 3D-4D region of interest (ROI) box determines the height and width of the volume acquired. The lateral dimensions of the ROI box have the greatest influence on frame rate. Therefore as a general rule, it is better to set the narrowest possible ROI that includes the cardiac apex and corresponding vertebral body. The width selection will depend on the focus of the examination. If it is the intention to obtain a volume that allows reconstruction of a complete segmental ap- proach, then the ROI box needs to be wide enough to encompass the stomach. However, if imaging is focused on the heart itself then the ROI box may be narrowed down to include both ventricles (fig. 4). This will provide the greater frame rate and may be preferable when additional imaging modalities such as color Doppler are added.

Acquisition Angle. The acquisition angle determines how many planes above and below the acquisition plane will be acquired. If a larger acquisition angle is chosen a larger portion of the fetus is sampled and the time to obtain this sample is therefore greater. While this does not affect the frame rate of the primary image, it renders the acquisition prone to movement artifact. Therefore, the acquisition angle should be adjusted to the area of interest. If a full segmental volume is to be acquired then the chosen angle needs to be large enough to encompass the neck all the way to the stomach. With a standard image in plane $\mathrm{A}$, as described above, the dimension of the box can be assessed in plane $\mathrm{B}$. The angle generally depends upon the size of the fetus. In the first and second trimesters, an angle of $20-25$ degrees is usually sufficient, while in the third trimester a 35-40 degree angle is often required to acquire a whole 'segmental' volume. During the acquisition, the operator will notice if the angle is adequate if he can visualize the stomach bubble at one end of the sweep and the 3-vessel view at the other.

Acquisition Time. The acquisition time is inversely proportional to the speed at which the mechanical sweep is performed. For a given acquisition angle a short acquisition time obtains fewer cross-sectional images and therefore has a lower image resolution, particularly in the $\mathrm{B}$ and $\mathrm{C}$ planes. Lengthening the acquisition time improves the resolution, but increases the chance of artifacts due to fetal movements. Typical acquisition times range between 7.5 and $15 \mathrm{~s}$. 

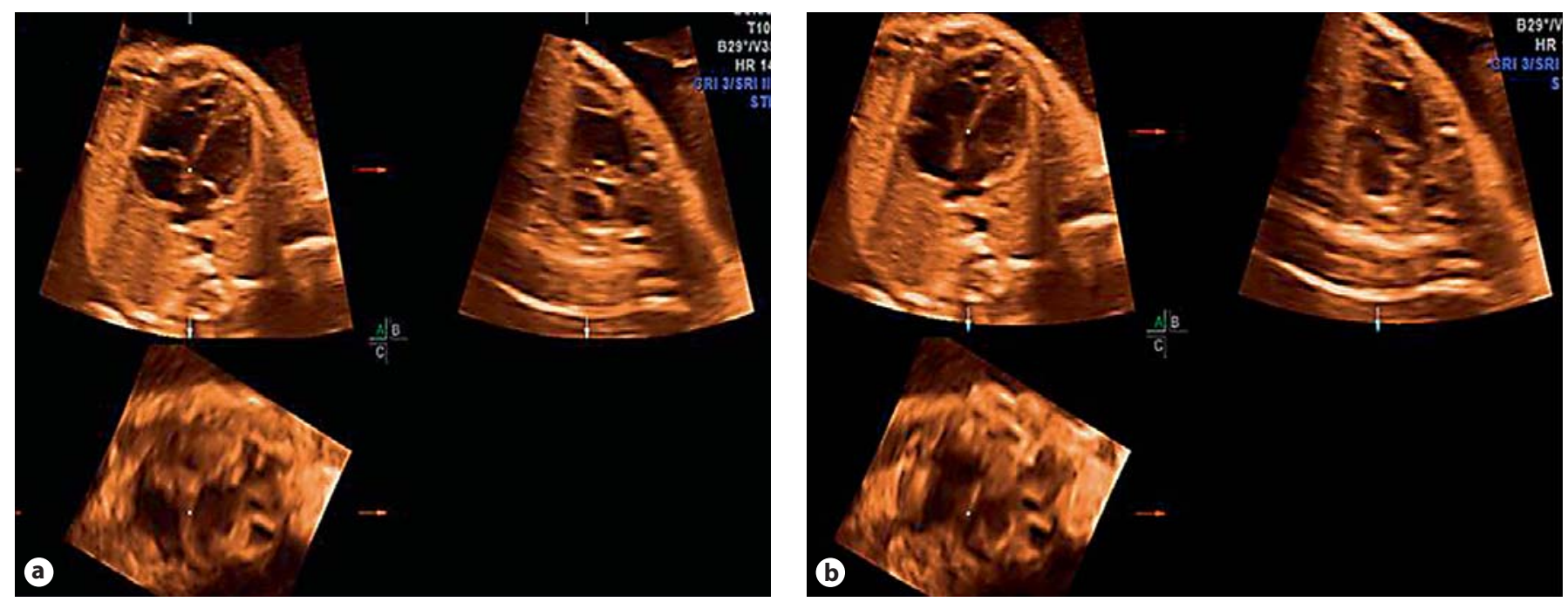

Fig. 5. Spatiotemporal image correlation acquisition in systole (a) and diastole (b) at the level of the 4-chamber view.

\section{D Motion Display}

Examining the fetal heart in motion is an important component of assessing cardiac structure and function. When displaying 3D image information as a moving image, the principal options are 3D cine-looping and 4D realtime imaging. When these modalities are chosen the spatial and temporal resolution of the image is limited. Motion artifacts can occur if the volume scan is relatively slow compared to rapid cardiac motion, and/or if the probe moves during the scan. Because the image acquisition and cardiac motion with the cardiac cycle are not synchronized, a simple cine-loop display of serial images may not display the motion of cardiac structures in an optimal way. In order to achieve the best anatomical display throughout the cardiac cycle, a fixed point of the image should be correlated spatially over a period of time. One of the most significant developments in fetal cardiac scanning is therefore Spatiotemporal image correlation (STIC) imaging.

\section{Spatiotemporal Image Correlation}

STIC is an automated volume acquisition in which a motion-gated offline analysis creates a cine-loop of optimally aligned 2D cross-sectional images. The transducer array performs a slow, single sweep, recording one single $3 \mathrm{D}$ data set over a 7.5-30 s time period. This volume of interest is acquired at a sweep angle of approximately 20 40 degrees (depending on the size of the fetus) and frame rate of about 150 frames per second. If a volume acquisi- tion time of $10 \mathrm{~s}$ and sweep area of 25 degrees are used, $1,500 \mathrm{~B}$-mode images are stored in memory. During this acquisition time the fetal heart beats 20-25 times, which means that within these $1,500 \mathrm{~B}$-mode frames there are 20-25 images showing systolic peaks. This information is used to calculate the fetal heart rate [7-10]. STIC does not identify specific cardiac structures but analyzes the rhythmic movement, independent of its direction, and it derives the heart rate from the periodicity of these movements. As a result, 40 consecutive volumes are used to reconstruct a complete heart cycle that is displayed in an endless loop. This cine-like file of a beating fetal heart can be manipulated to display any acquired scanning plane at any stage in the cardiac cycle and calculates average heart rate (fig. 5) [11]. If there are abrupt changes in the heart rate during acquisition the STIC algorithm has difficulties in calculating the average heart rate correctly, and this will lead to artifacts due to rearrangement of non-corresponding images. In these circumstances repeat acquisition within a short period of time (7.5-15 s) should be performed. Optimal STIC acquisition for examination of the fetal heart has been described by Goncalves et al. [9]. In post-processing, the cardiac volumes are displayed as one real-time cardiac cycle, played in a cine-loop. The loop may also be played in slow motion, stopped and analyzed any time for detailed analysis of specific phases of the cardiac cycle. Classic and virtual planes that are not accessible with 2D scanning can be obtained and saved for expert review or educational purposes [12-14]. 


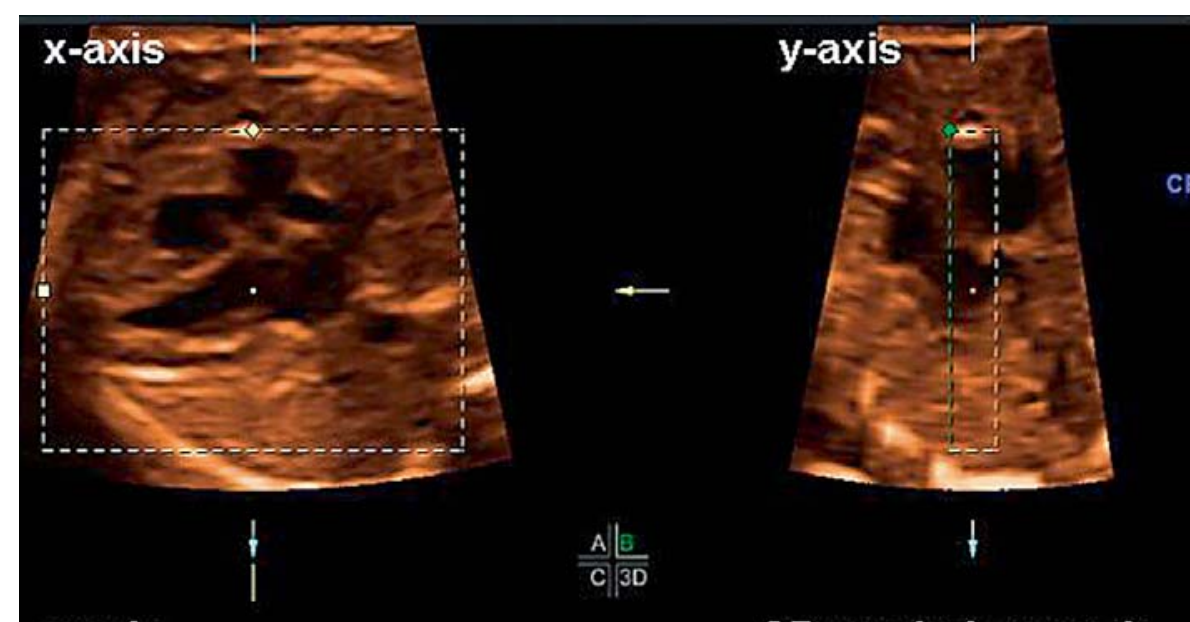

Fig. 6. $3 \mathrm{D}$ multiplanar reconstruction at the level of the 4-chamber view. The image plane can be manipulated in the $\mathrm{x}, \mathrm{y}$ and $\mathrm{z}$ axes. The 3D image demonstrates the type of rendering mode. The navigation point is placed close to the left ventricle in the A plane, the B plane shows the septum 'en face' and the $\mathrm{C}$ plane shows the coronal plane through the ventricles.

\section{z-axis}

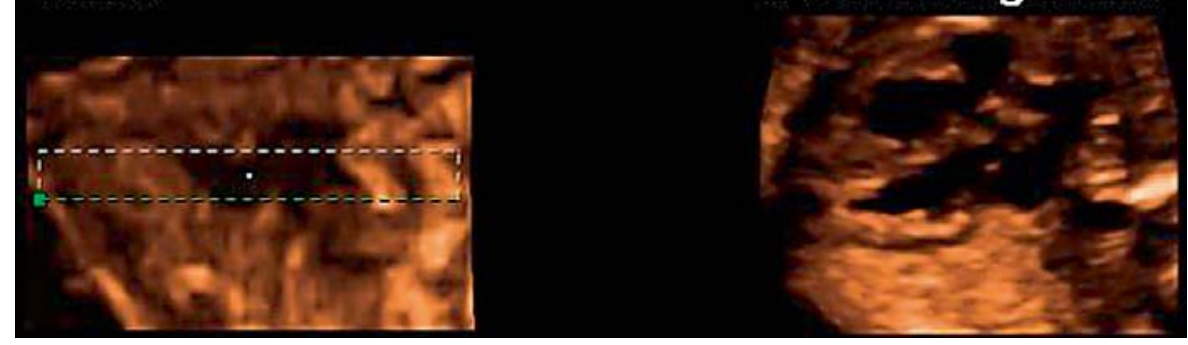

\section{D Post-Processing Possibilities}

Once a 3D volume is acquired, several post-processing techniques are available that facilitate the display of certain anatomic aspects. Rendering is an algorithm that, by simulation of lights and shadows, allows the representation of a $3 \mathrm{D}$ object through a $2 \mathrm{D}$ image. The difference between a rendered and a $2 \mathrm{D}$ image is the depth that is projected into the $\mathrm{z}$ axis. The operator places a box at the level of the ROI within the volume to show a slice of the volume whose depth reflects the thickness of the slice (fig. 6).

Surface Rendering. Using a low level of transparency, a surface rendering mode can be applied to the fetal heart using the interface between the cavities and the cardiac walls. It allows the operator to obtain cardiac views not otherwise available with 2D ultrasound (fig. 7a).

Minimum Mode Rendering. Increasing transparency to a high level enables the heart and blood vessels to be seen in a projection. By choosing this mode, structures with high transparency (anechoic) can be demonstrated as a $3 \mathrm{D}$ projection of black on a background of additional imaging information (fig. $7 \mathrm{~b}$ ).
Transverse Rendering. Occurs in the B plane where an image is rendered from 'front to back'. This process saves the step of changing render direction (fig. 7c).

Inversion Mode. Inversion mode (IM) is another postprocessing visualization modality that can be combined with static 3D or STIC acquisition. IM analyzes the echogenicity of tissue (white) and fluid-filled areas (black) in a volume and inverts their presentation. Fluid-filled spaces such as the cardiac chambers then appear white, while the myocardium disappears (fig. 7d). In fetal echocardiography it can be applied to create digital casts of cardiac chambers and vessels. It can also produce a reconstruction of the extra-cardiac vascular tree, similar to 3D power Doppler. IM has the additional advantage of showing the stomach and gall bladder as white structures, which can aid the operator in navigating within a complex scan. Most recently, IM has been joined with STIC to quantify fetal cardiac ventricular volumes and cardiac output, which may prove useful in the evaluation of fetal heart function [15].

Glass Body. The volume is acquired with color or power Doppler. The acquisition can be a static $3 \mathrm{D}$ volume or a STIC volume, and it can be displayed in 3 modalities: either in grayscale alone, in color scale alone, or in a combination 

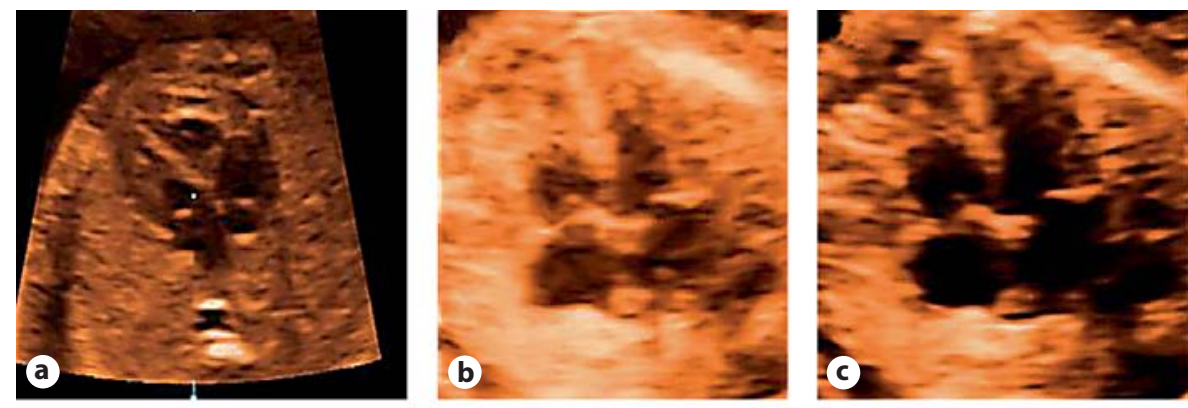

Fig. 7. Different rendering modalities representation of 4 -chamber view. a Surface rendering. b Minumum mode. c Transverse mode. d Inversion mode. e Glass body.
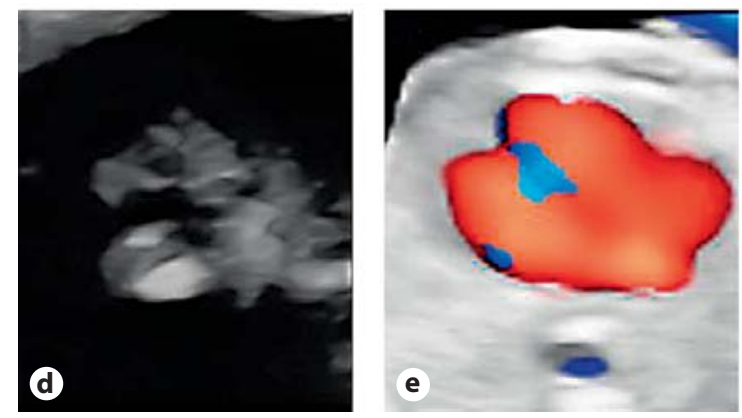

of both (glass body mode). This rendering algorithm is based on the simultaneous representation of gray and color Doppler scale, and it allows demonstration, at the same time, of the ventricular filling and the crossing of the great arteries, without changing the scanning plane (fig. 7e).

\section{Multiplanar Imaging Techniques}

Once a 3D volume is obtained in any of the acquisition modes, the ultrasound information in any of the planes, can be displayed in an automated way. In the multiplanar reconstruction mode (MPR) the intersection of the 3 planes is presented as a dot and 3 perpendicular planes and the volume model in the fourth frame of the image are displayed (fig. 6). The reference dot guides the operator during navigation within the volume. While the $\mathrm{A}, \mathrm{B}$ and $\mathrm{C}$ planes are important to standardize the primary imaging axes within the volume, they are not very practical to study anatomic relationships. Adapted from computerized tomography and magnetic resonance imaging interpretation, automated display of parallel imaging planes is an intuitive method to analyze anatomical relationships. Automated display of adjacent imaging planes of a $3 \mathrm{D}$ volume sample is available using a generic parallel arrangement with variable slice thickness, or modified arrangements in imaging planes that account for the physiologic anatomic orientation of the traditional car- diac imaging planes. The former technique is known as tomographic ultrasound imaging (TUI), while the latter is known as volume computer-aided diagnosis (VCAD).

Cardiac volumes are represented as a cardiac cycle in real time, and can be reproduced in a clip. The clip can be displayed using a variety of imaging modalities and at various play speeds. Images can be stopped to perform detailed analyses of specific phases of the cardiac cycle. These techniques open many possibilities for clinical applications.

\section{Tomographic Ultrasound Imaging}

TUI is an automated display modality where parallel images in a $3 \mathrm{D}$ volume are displayed in a multi-image display. TUI allows the examiner to simultaneously display multiple cross-sectional images at specific distances from the 4-chamber view [16]. The number of the slices and distances between the slices can be adjusted by the operator. The upper left frame of the display shows the position of each plane within the ROI, relative to the reference plane. In order to achieve a standardized display, the reference image needs to be positioned in a standardized way as described above. Because the display is strictly parallel, the positioning of the reference dot does not affect the multi-image display. The advantage of this application is the ability to display sequential parallel planes simultaneously giving a more complete picture of the fetal heart (fig. 8). 


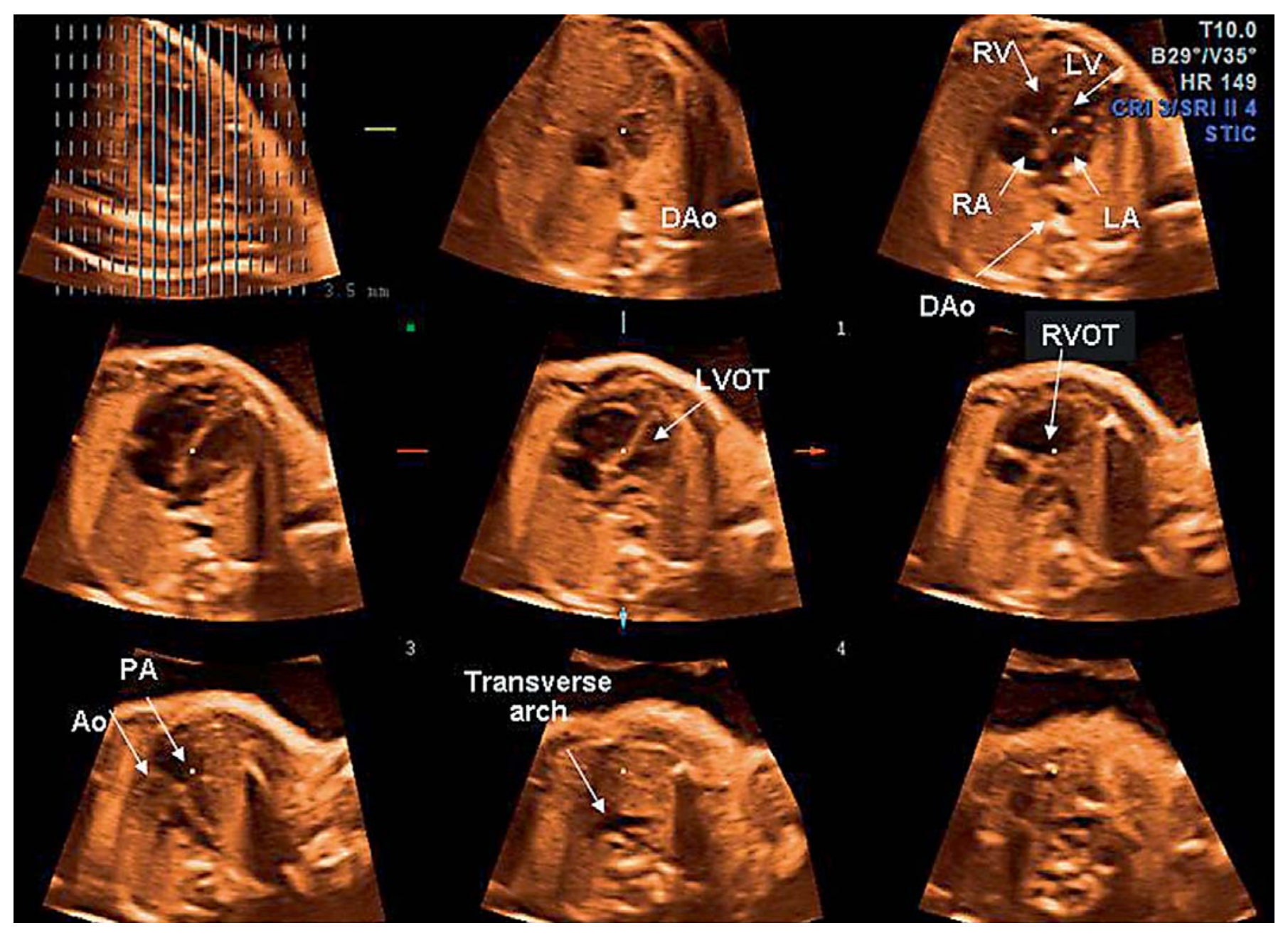

Fig. 8. TUI mode. Simultaneous visualization of transverse planes starting from the abdomen up to the outflow tracts. Ao = Aorta; DAo = descending aorta; $L A=$ left atrium; $L V=$ left ventricle; $L V O T=$ left ventricle outflow tract; $\mathrm{PA}=$ pulmonary artery; $\mathrm{RA}=$ right atrium; $\mathrm{RV}=$ right ventricle; $\mathrm{RVOT}=$ right ventricle outflow tract.

\section{Volume Computer-Aided Diagnosis}

VCAD, utilizing formulas published by Abuhamad et al. [17], is intended for use between 18 and 23 weeks of gestation. Starting from the 4-chamber view, a volume of the entire fetal chest from the stomach to the neck is acquired. Sweep angles typically vary between 30 and 45 degrees. The volume acquisition can be accomplished by using either the 3D static or the STIC modes. Once the acquisition is completed, the transverse image of the chest containing the 4 -chamber view of the heart is rotated so that the spine is at 6 o'clock and the apex of the heart is between 10 and 11 o'clock in the A plane (fig. 9, top left image). The dot, which indicates the intersection of the 3 planes, should be positioned over the crux of the heart, between the insertions of the AV valves. In the $\mathrm{B}$ plane, the spine should be horizontal (fig. 9, top right image). Once the 4-chamber view is oriented as described above, the VCAD system can help to display the left outflow tract, the right outflow tract, and the position of the stomach in the abdomen. All the anatomical views are displayed in the TUI mode, which displays a number of parallel slices through the volume data set. Applying the TUI feature adds to the outcome and the reliability of VCAD since the desired view is consistently shown in one of the planes. In contrast to the simple TUI technique, VCAD also requires a standardized placement of the reference dot, because the displayed planes also account for the angulation required to provide an optimal display of the outflow tracts. 
Fig. 9. Volume computer-aided diagnosis. The reference image is oriented in a standardized way. A plane, top left image: spine is at 6 o'clock, apex of the heart between 10 and 11 o'clock. B plane, top right image: spine is horizontal.

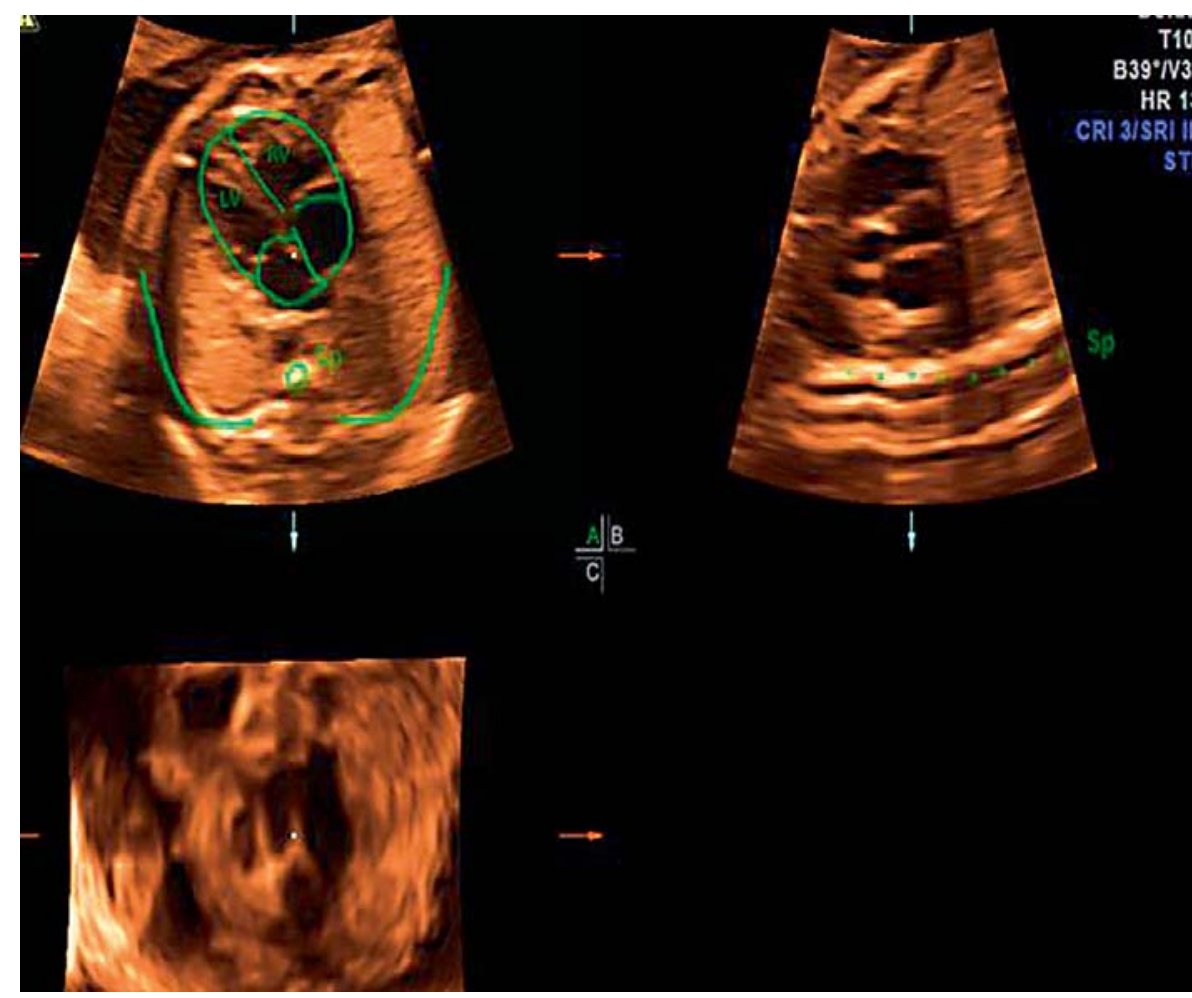

\section{Scrolling, Spin and TUI Technique}

This modality is based on cranial and caudal scroll starting from the 4-chamber view without using rotations, and it allows visualization of situs, left ventricular outflow tract, right ventricular outflow tract and the 3-vessel view. Starting from the acquired volume, the 4 -chamber view is optimized modifying resolution, zoom and the clip velocity (between 25 and $50 \%$ of the original fetal heart rate). Moving the $y$ axis in plane $B$ from left to right or vice ver$\mathrm{sa}$, it is possible to check the situs and the position of the stomach and the left ventricular outflow tract. Further cranially, it is possible to visualize the pulmonary artery, the 3-vessel view with thymus, and trachea. Spin technique is based on different rotations (spin) on $\mathrm{y}$ and $\mathrm{z}$ axis to visualize the outflow tracts (fig. 10a-f).

\section{Fetal Cardiac Volume Analysis}

Automated calculation algorithms can be used to calculate the volumes of the cardiac chambers by tracing the interface between the chamber walls and cavities. These techniques have great applications in the evaluation of cardiac function.

\section{Virtual Organ Computer-Aided Analysis}

This semi-automated volume measurement tool known by the acronym VOCAL - utilizes computer technology to provide accurate volume calculations. The acquisition can be a static $3 \mathrm{D}$ volume or a STIC volume in traditional or inversion modes. Once the volume is acquired the wall-cavity interface is traced in a number of planes. The number of planes is determined by the degrees of separation between them. Typically a 12-degree separation provides a sufficiently reproducible volume calculation. The acquired volumes allow quantification of stroke volume and ejection fraction [18].

\section{D Fetal Echocardiography in Clinical Applications}

Several developments of 3D imaging techniques facilitate the application in clinical settings. These include automated retrieval of anatomically relevant imaging planes and the ability of motion display modalities which offer comparable reviewing possibilities similar to traditional video recordings with the significant benefit of image manipulation by the reviewer. The application of these techniques has been evaluated in a low-risk 

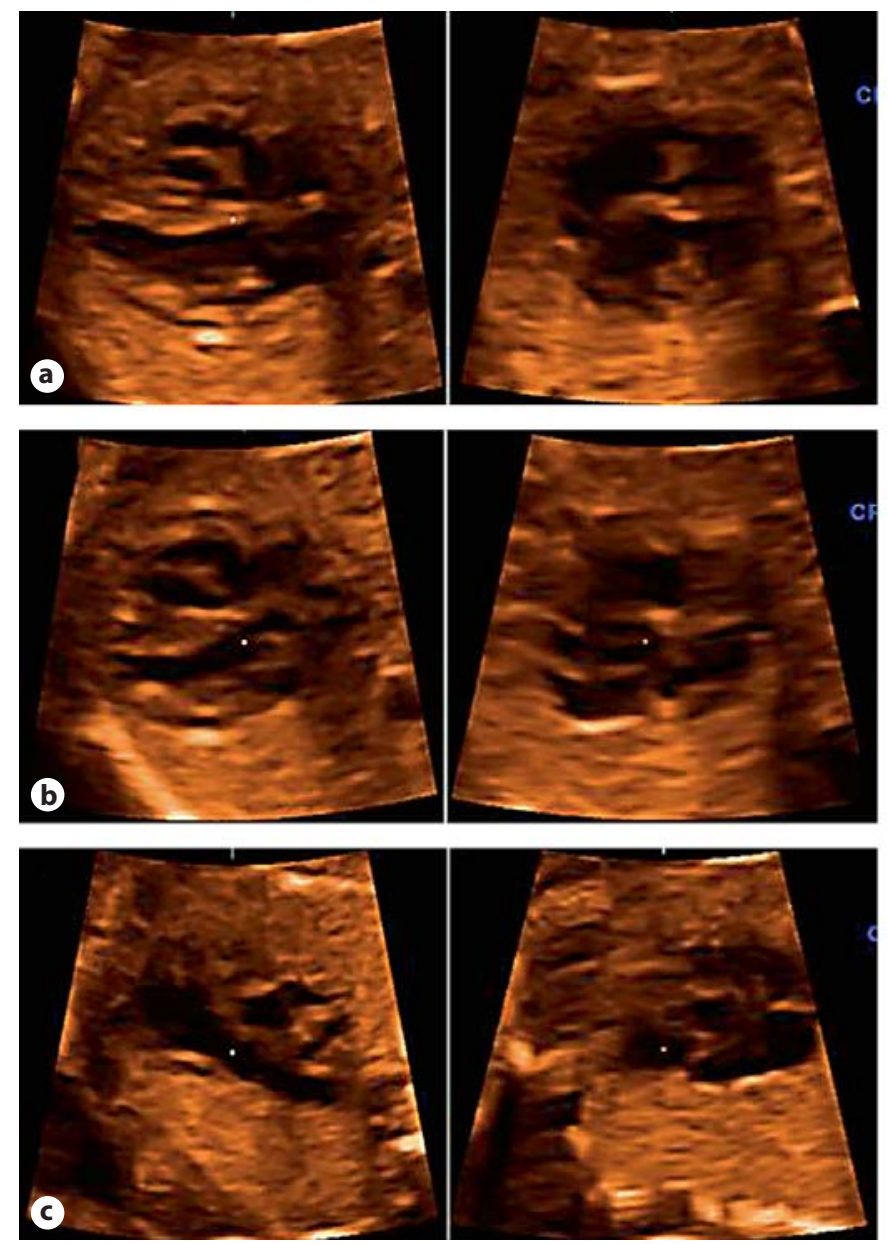

Fig. 10. After optimization of the 4-chamber view, the cardiac apex is placed at 9 o'clock (a), scrolling cranially on the A plane allows visualization of the left ventricular outflow tract (b), scrolling more on the A plane demonstrates the right ventricular outflow tract (c) and the 3 -vessel view. Moving the reference point

screening context as well as the diagnosis of cardiac anomalies.

Performance of STIC in a Cardiac Screening Program. The advantages that have been cited for this technique are that it allows review of an unlimited number of images, correlation of images that are perpendicular to acquisition plane, storage capability and volume assessment by the examiner or by experts at a remote site. With this in mind, the question of 'Can this technique be widely used for screening purposes in specialist centers and remote areas?' still remains unclear. Several studies have tried to answer this question. Chaoui et al. [19] were able to obtain cardiac volumes by STIC in $95 \%$ of cases in tertiary centers, with $70-90 \%$ adequate visualization of the fetal heart
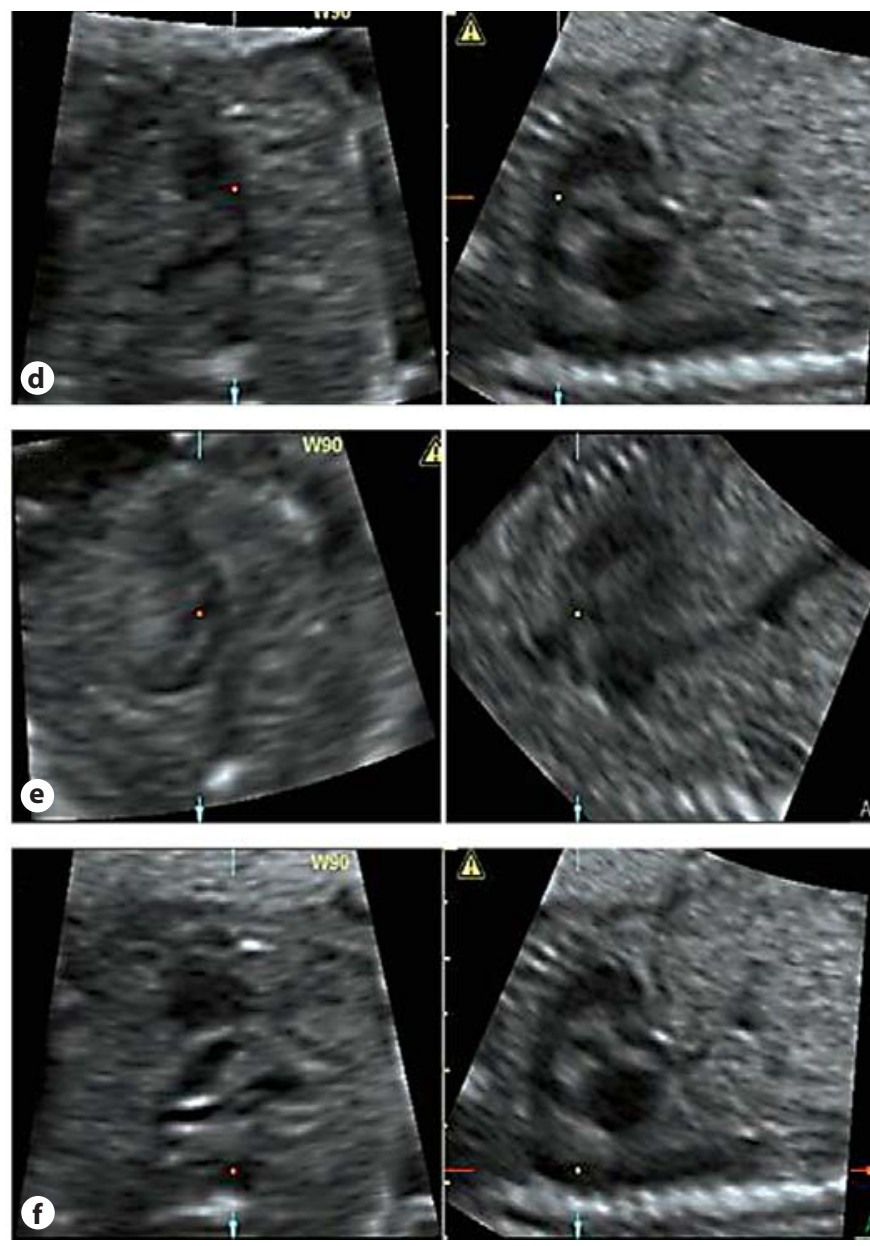

inside the pulmonary and rotating on the $\mathrm{z}$ axis to align the $\mathrm{y}$ axis with the pulmonary one can display the ductal arch (d). In a similar way, moving the reference point to the aorta and descending aorta by aligning the $\mathrm{y}$ axis in plane $\mathrm{A}$, one can demonstrate the aortic arch (e) and descending aorta (f). being reported by Rizzo and coworkers [20]. STIC is used mainly in specialist centers as it requires ultrasound machines capable of performing the technique and analyzing the volumes acquired, as well as the software to analyze the volume offline. Viñals et al. [21] demonstrated that it was possible for a general obstetrician familiar with ultrasound imaging to acquire STIC volume $100 \%$ of the time at gestational ages between 18 and 37 weeks. A 94-96\% success rate in the visualization of cardiac landmarks was reported. They concluded that it was feasible for images that were acquired by a generalist to be analyzed by a practitioner with special experience in fetal echocardiography and that prenatal exclusion of cardiac abnormalities was feasible. The same group also performed a pilot study an- 
alyzing the feasibility of using STIC telemedicine via internet link with general obstetricians in a remote part of Chile. The operators were using equipment capable of $4 \mathrm{D}$ ultrasound with STIC, but they did not have training in detailed fetal echocardiography. They were taught the technique of volume acquisition by e-mail and a single experienced investigator analyzed the acquired images via Internet link. In 50 patients, a full cardiac examination could be completed on $85-96 \%$ of the volumes [22]. The study was repeated in 2008 for 35 fetuses at 11 to 13 +6 weeks' gestation. The acquisition of adequate volumes was more difficult due to fetal movement but it was possible to obtain and analyze complete volume datasets when the fetuses were beyond 12 weeks' gestation [23]. Our group also standardized the technique for examination at the first trimester using STIC and TUI in combination. The advantage of adding the TUI application is the ability to adjust slice thickness below $1 \mathrm{~mm}$, which allows display of standard cardiac examination planes with appropriate definition and precision and identification of all cardiac landmarks in $85 \%$ of low-risk patients [24]. In another study, Uittenbogaard et al. [39] demonstrated that STIC volumes could be acquired by sonographers within a time slot allocated for echocardiography examination in $76 \%$ of cases. They concluded that sonographers do not have to be specifically experienced in $3 \mathrm{D}$ and $4 \mathrm{D}$ ultrasound imaging to acquire high-quality STIC volumes. Another study, from Paladini et al. [26], assessed if medically qualified sonologists with low to intermediate experience of anomaly ultrasound screening in the second trimester and no experience of insonating the outflow tracts are able to detect major abnormalities of the outflow tracts by reviewing the A plane of cardiac volume datasets acquired with STIC volume. After a 2-hour lecture on abnormal outflow tract views and the use of the software, 14 sonographers had an individual diagnostic accuracy ranging form $66-100 \%$ [26].

These studies suggest that acquisition of quality STIC volumes does not require expert skills in fetal cardiac imaging. This is an important prerequisite to allow mass screening with off-site expert analysis. Population-based performance of such a cardiac screening program and the evaluation of the logistics require further study.

Performance of STIC in Patients with Cardiac Abnormalities. Characterization of complex congenital heart disease is particularly challenging in the prenatal period because of image resolution, variability of insonation possibilities and skills required by the examiner. Several authors have shown in the past that despite these difficulties a detailed diagnosis of congenital heart disease with good level of accuracy is possible in fetuses [27, 28]. The application of STIC technology in combination with other modalities appears to be helpful in understanding of cardiac anatomy, vascular development and cardiac function.

Yagel et al. [29] demonstrated that rendering STIC technology allows the visualization of virtual planes such as the inter-ventricular and inter-atrial septa as well as the atrioventricular annular plane just distal to the semiulnar valves and foramen ovale. They analyzed 136 normal and 35 abnormal cases and concluded that rendering STIC technology allows better visualization of these virtual planes and improved rates of diagnosis of atrial and ventricle septal defects compared to $2 \mathrm{D}$ ultrasound [29]. In addition, this imaging information may also aid postpartum surgical correction, as shown by Jouannic et al. [30], who studied the impact of prenatal diagnosis on neonatal clinical status in patients with transportation of the great arteries. They found that a restrictive foramen ovale and/or ductus arteriosus predicts the need for emergency intervention after birth.

The role of STIC with TUI for the simultaneous display of sequential cardiac examination planes in the analysis of fetal congenital heart disease was demonstrated by Paladini et al. [31]. The same group also reported the benefits of using STIC with color Doppler. They reported that it was possible to define the spatial relationships between the great arteries in fetuses with transposition of the great arteries with a high degree of accuracy [32]. The combination of STIC with color Doppler in 3 imaging planes (4chamber, 5-chamber and 3-vessel tracheal view) was also studied by Chaoui et al. [33]. This approach was successful in 24 of 27 fetuses with cardiac anomalies and allowed a reviewer at a remote site to visualize the abnormality using at least 1 of the 3 planes [33]. The same group also used 3D power Doppler ultrasonography to demonstrate the spatial presentation of fetal vessels in utero. In their study they were able to show that a 3D demonstration of fetal vessels is possible with the system used. Display accuracy was limited by fetal movements and unfavorable fetal positioning, but the possible benefit of this method to diagnose complex fetal vascular malformations was noted [34]. Our group also studied the feasibility of STIC and 3D power Doppler for detailed definition of aortic arch abnormalities. Our study confirms that the upper thoracic 3 -vessel view is an effective 2D echocardiographic screening plane to identify isolated aortic arch abnormalities. However, 3D echocardiography with grayscale and color Doppler STIC was superior in identifying the precise vascular anatomy [6]. The combination of B-flow and STIC has superior ability for imaging of very small vessels such 
as the pulmonary veins $[35,36]$ and may prove superior in patients with venous anomalies.

The use of STIC in combination with inversion mode and VOCAL for estimation of fetal cardiac volume has been demonstrated in several studies $[37,38]$. The ability to measure ejection fraction and stroke volume of these techniques is very helpful, especially in congenital cardiac malformations, hydrops, fetal diabetic cardiomyopathy, and intrauterine growth restriction or twin-to-twin transfusion syndrome. The information may prove valuable for making diagnoses and arranging intervals for fetal surveillance [39].

\section{Conclusions}

The image quality, post-processing possibilities, automated display of anatomically relevant imaging planes and the motion display of cardiac structures that incor- porates all of these possibilities has reached a new level of sophistication with modern ultrasound equipment. The feasibility and reproducibility of these imaging technologies for 3D/4D fetal echocardiography has been demonstrated. Future research will clarify how these techniques are best utilized for fetal cardiac screening programs and the detailed evaluation of congenital cardiac disease. The incorporation of dedicated vascular imaging modality specific applications for complex vascular anomalies is likely to emerge. The capabilities for functional cardiac assessment may allow new insights into pathophysiology of anatomic or functional cardiac conditions. The nondestructive unlimited editing possibilities of stored image information will accelerate training of sonographers and sonologists. Three-dimensional echocardiography is already an indispensable diagnostic and management tool in many fetal therapy centers across the world and its role is likely to expand in the future.

\section{References}

$\checkmark 1$ Henry WL, DeMaria A, Gramiak R, King DL, Kisslo JA, Popp RL, Sahn DJ, Schiller NB, Tajik A, Teichholz LE, Weiman AE: Report of the American Society of Echocardiography Committee on nomenclature and standards in two-dimensional echocardiography. Circulation 1980;62:212-217.

$\checkmark 2$ Allan L: Antenatal diagnosis of heart disease. Heart 2000;83:367.

-3 Yagel S, Cohen SM, Achiron R: Examination of the fetal heart by five short-axis views: a proposed screening method for comprehensive cardiac evaluation. Ultrasound Obstet Gynecol 2001;17:367-369.

4 Carvalho JS, Ho SY, Shinebourne EA: Sequential segmental analysis in complex fetal cardiac abnormalities: a logical approach to diagnosis. Ultrasound Obstet Gynecol 2005; 26:105-111.

$>5$ Schiky-Tamir Y, Cohen SM, Hochner-Celnikier D, Valsky DV, Messing B, Yagel S: Threedimensional power Doppler (3 DPD) ultrasound in the diagnosis and follow-up fetal vascular anomalies. Am J Obstet Gynecol 2006;194:274-281.

-6 Turan S, Turan OM, Maisel P, Gaskin P, Harman CR, Baschat AA: Three-dimensional sonography in the prenatal diagnosis of aortic arch abnormalities. J Clin Ultrasound 2009;37:253-257.

$>7$ DeVore GR, Falkensammer P, Sklansky MS, Platt LD: Spatiotemporal image correlation (STIC): new technology for evaluation of the fetal heart. Ultrasound Obstet Gynecol 2003; 22:380-387.
$>8$ Deng J: Terminology of three-dimensional and four-dimensional ultrasound imaging of the fetal heart and other moving body parts. Ultrasound Obstet Gynecol 2003;22: 336-344.

$\checkmark 9$ Goncalves LF, Lee W, Chaiworapongsa T, Espinoza J, Schoen ML, Falkensammer P, Treadwell M, Romero R: Four dimensional ultrasonography of the fetal heart with spatiotemporal image correlation. Am J Obstet Gynecol 2003;189:1792-1802.

10 Falkensammer P: Spatio-Temporal Image Correlation for Volume Ultrasound: Studies of the Fetal Heart. Zipf, GE Healthcare, 2000.

11 Yagel S, Cohen SM, Shapiro I, Valsky DV: 3D and 4D ultrasound in fetal cardiac scanning: a new look at the fetal heart. Ultrasound $\mathrm{Ob}$ stet Gynecol 2007;29:81-95.

12 Yagel S, Arbel R, Anteby EY, Raveh D, Achiron $\mathrm{R}$ : The three vessels and trachea view $(3 \mathrm{VT})$ in fetal cardiac scanning. Ultrasound Obstet Gynecol 2002;20:340-345.

13 DeVore GR, Polanco B, Sklansky MS, Platt LD: The 'spin' technique: a new method for examination of the fetal outflow tracts using three-dimensional ultrasound. Ultrasound Obstet Gynecol 2004;24:72-82.

14 Abuhamad A: Automated multiplanar imaging: a novel approach to ultrasonography. J Ultrasound Med 2004;23:573-576.
15 Messing B, Cohen SM, Valsky DV, Rosenak D, Hochner-Celnikier D, Savchev S, Yagel S: Fetal cardiac ventricle volumetry in the second half of gestation assessed by 4D ultrasound using STIC combined with inversion mode. Ultrasound Obstet Gynecol 2007;30: 142-151.

16 Palladini D, Vassallo M, Sglavo G, Lapadula C, Martinelli P: The role of spatiotemporal image correlation (STIC) with tomographic ultrasound imaging (TUI) in the sequential analysis of fetal congenital heart diseases. Ultrasound Obstet Gynecol 2006;27:555561.

17 Abuhamad A, Falkensammer P, Reichartseder F, Zhao Y: Automated retrieval of standard diagnostic fetal cardiac ultrasound planes in the second trimester of pregnancy: a prospective evaluation of software. Ultrasound Obstet Gynecol 2008;31:30-36.

18 Yagel S, Cohen SM, Shapiro I, Valsky DV: Fetal cardiac ventricle volumetry in the second half of gestation assessed by 4D ultrasound using STIC combined with inversion mode. Ultrasound Obstet Gynecol 2007;30:142151.

19 Chaoui R, Hoffmann J, Heling KS: Three-dimensional (3D) and 4D color Doppler fetal echocardiography using spatio-temporal image correlation (STIC). Ultrasound Obstet Gynecol 2004;23:535-545. 
20 Rizzo G, Capponi A, Muscatello A, Cavicchioni O, Vendola M, Arduini D: Examination of the fetal heart by four-dimensional ultrasound with spatiotemporal image correlation during routine second-trimester examination: the 'three-steps technique'. Fetal Diagn Ther 2008;24:126-131.

-21 Viñals F, Poblete P, Giuliano A: Spatio-temporal image correlation (STIC): a new tool for the prenatal screening of congenital heart defects. Ultrasound Obstet Gynecol 2003;22: 388-394.

-22 Viñals F, Mandujano L, Vargas G, Giuliano A: Prenatal diagnosis of congenital heart disease using four-dimensional spatio-temporal image correlation (STIC) telemedicine via an Internet link: a pilot study. Ultrasound Obstet Gynecol 2005;25:25-31.

-23 Viñals F, Ascenzo R, Naveas R, Huggon I, Giuliano A: Fetal echocardiography at $11+0$ to $13+6$ weeks using four-dimensional spatiotemporal image correlation telemedicine via an Internet link: a pilot study. Ultrasound Obstet Gynecol 2008;31:633-638.

24 Turan S, Turan OM, Ty-Torredes K, Harman CR, Baschat AA: Standardization of the first-trimester fetal cardiac examination using spatiotemporal image correlation with tomographic ultrasound and color Doppler imaging. Ultrasound Obstet Gynecol 2009; 33:652-656.

-25 Viñals F, Ascenzo R, Naveas R, Huggon I, Giuliano A: A systematic analysis of the feasibility of four-dimensional ultrasound imaging using spatiotemporal image correlation in routine fetal echocardiography. Ultrasound Obstet Gynecol 2008;31:625632.

26 Paladini D, Sglavo G, Greco E, Nappi C: Cardiac screening by STIC: can sonologists performing the 20-week anomaly scan pick up outflow tract abnormalities by scrolling the A-plane of STIC volumes? Ultrasound Obstet Gynecol 2008;32:865-870.
27 Allan LD, Sharland GK, Milburn A, Lockhart SM, Groves AM, Anderson RH, Cook AC, Fagg NL: Prospective diagnosis of 1,006 consecutive cases of congenital heart disease in the fetus. J Am Coll Cardiol 1994;23:14521458.

28 Paladini D, Russo M, Teodoro A, Pacileo G, Capozzi G, Martinelli P, Nappi C, Calabrò R: Prenatal diagnosis of congenital heart disease in the Naples area during the years 1994-1999: the experience of a joint fetal-pediatric cardiology unit. Prenat Diagn 2002; 22:545-552.

29 Yagel S, Benachi A, Bonnet D, Dumez Y, Hochner-Celnikier D, Cohen SM, Valsky DV, Fermont L: Rendering in fetal cardiac scanning: the intracardiac septa and the coronal atrioventricular valve planes. Ultrasound Obstet Gynecol 2006;28:266-274.

-30 Jouannic JM, Gavard L, Fermont L, Le Bidois J, Parat S, Vouhé PR, Dumez Y, Sidi D, Bonnet D: Sensitivity and specificity of prenatal features of physiological shunts to predict neonatal clinical status in transposition of the great arteries. Circulation 2004; 110: 1743-1746.

- 31 Paladini D, Vassallo M, Sglavo G, Lapadula C, Martinelli P: The role of spatio-temporal image correlation (STIC) with tomographic ultrasound imaging (TUI) in the sequential analysis of fetal congenital heart disease. Ultrasound Obstet Gynecol 2006;27:555-561.

32 Paladini D, Volpe P, Sglavo G, Vassallo M, De Robertis V, Marasini M, Russo MG: Transposition of the great arteries in the fetus: assessment of the spatial relationships of the arterial trunks by four-dimensional echocardiography. Ultrasound Obstet Gynecol 2008;31:271-276.

33 Chaoui R, Hoffmann J, Heling KS: Three-dimensional (3D) and 4D color Doppler fetal echocardiography using spatio-temporal image correlation (STIC). Ultrasound $\mathrm{Ob}$ stet Gynecol 2004;23:535-545.
34 Hartung J, Kalache KD, Chaoui R: Three-dimensional power Doppler ultrasonography (3D-PDU) in fetal diagnosis (article in German, abstract in English). Ultraschall Med 2004;25:200-205.

35 Volpe P, Campobasso G, De Robertis V, Di Paolo S, Caruso G, Stanziano A, Volpe N, Gentile M: Two- and four-dimensional echocardiography with B-flow imaging and spatiotemporal image correlation in prenatal diagnosis of isolated total anomalous pulmonary venous connection. Ultrasound $\mathrm{Ob}$ stet Gynecol 2007;30:830-837. Erratum: Ultrasound Obstet Gynecol 2008;31:365.

- 36 Volpe P, Campobasso G, Stanziano A, De Robertis V, Di Paolo S, Caruso G, Volpe N, Gentile M: Novel application of 4D sonography with B-flow imaging and spatio-temporal image correlation (STIC) in the assessment of the anatomy of pulmonary arteries in fetuses with pulmonary atresia and ventricular septal defect. Ultrasound Obstet Gynecol 2006;28:40-46.

37 Messing B, Cohen SM, Valsky DV, Rosenak D, Hochner-Celnikier D, Savchev S, Yagel S: Fetal cardiac ventricle volumetry in the second half of gestation assessed by $4 \mathrm{D}$ ultrasound using STIC combined with inversion mode. Ultrasound Obstet Gynecol 2007;30: 142-151.

38 Molina FS, Faro C, Sotiriadis A, Dagklis T, Nicolaides KH: Heart stroke volume and cardiac output by four-dimensional ultrasound in normal fetuses. Ultrasound Obstet Gynecol 2008;32:181-187.

39 Uittenbogaard LB, Haak MC, Spreeuwenberg MD, van Vugt JM: Fetal cardiac function assessed with four-dimensional ultrasound imaging using spatiotemporal image correlation. Ultrasound Obstet Gynecol 2009;33:272-281. 